\title{
Les agro-solvants pour l'extraction des huiles végétales issues de graines oléagineuses
}

\author{
Frederic Fine ${ }^{1, a}$, Maryline Abert Vian ${ }^{2}$, Anne-Sylvie Fabiano Tixier ${ }^{2}$, Patrick Carre ${ }^{3}$, \\ Xavier Pages ${ }^{4}$ et Farid Chemat ${ }^{2}$ \\ 1 CETIOM, 11 rue Monge, 33600 Pessac, France \\ 2 Université d'Avignon et des Pays de Vaucluse, INRA, UMR408, 84000 Avignon, France \\ 3 CREOL, 11 rue Monge, 33600 Pessac, France \\ ${ }^{4}$ ITERG, 11 rue Monge, 33600 Pessac, France
}

Reçu le 28 novembre 2012 - Accepté le 28 mars 2013

\begin{abstract}
Résumé - Les huiles représentent une part importante du régime alimentaire chez l'homme et contiennent de nombreux nutriments essentiels. Les consommateurs se montrent de plus en plus attentifs aux mentions relatives à l'origine et à la qualité des produits qu'ils achètent. Quant aux industries, elles ont compris qu'il n'est désormais plus possible de produire sans penser à l'environnement. Cet article traite de l'utilisation d'agro-solvants afin de substituer l'hexane, solvant le plus largement utilisé pour l'extraction de graines oléagineuses. En effet, l'hexane présente de nombreux inconvénients, comme par exemple sa grande inflammabilité, sa dangerosité pour la santé et l'environnement et son origine non-renouvelable. Son utilisation dans le domaine de l'extraction est discutée depuis les années 1980, avec une intensité renouvelée depuis les années 2000. Une vue d'ensemble d'agro-solvants alternatifs est présentée.
\end{abstract}

Mots clés : Extraction d'huile / graine oléagineuses / agro-solvant, hexane

\begin{abstract}
Agro-solvents for the oilseed extraction. Oils represent an important part in human diet and contain essential nutriments. Consumers are more and more swayed by the argument of product origin. As for industries, they understood it's not possible anymore to produce without thinking about the environment. This article deals with the use of agro-solvents to substitute hexane which is the most widely used solvent in oilseed extraction. Indeed, hexane presents numerous drawbacks like high flammability, dangerousness for health and environment and a non-renewable origin. Its use in the field of extraction is discussed since the $80 \mathrm{~s}$ with a renewed intensity since the 00s. An overview of alternative solvents was presented.
\end{abstract}

Keywords: Oil extraction / oilseed / agro-solvent / hexane

\section{Introduction}

Malgré quelques évolutions techniques visant à son optimisation et à une meilleure maîtrise, le procédé industriel d'extraction des huiles oléagineuses n'a que peu évolué au cours des cinquante dernières années. Il correspond à la succession des opérations unitaires suivantes (Fig. 1) :

- préparation des graines : nettoyage, décorticage, précuisson, aplatissage ;

- cuisson : les flocons issus des étapes précédentes subissent une cuisson à la vapeur indirecte ;

- pression : des presses continues exercent une pression sur les graines; le tourteau ainsi obtenu, appelé «gâteau de pression ", contient encore de 16 à $24 \%$ de matières grasses selon la nature des graines traitées ;

- extraction de l'huile du «gâteau de pression » à l'aide d'un extracteur continu à percolation par solubilisation de l'huile dans l'hexane;

\footnotetext{
${ }^{a}$ Correspondance : fine@acetiom. fr
}

- désolvantation du tourteau par désorption et évaporation de l'hexane;

- distillation de l'huile pour l'évaporation du solvant.

Les huiles végétales brutes résultant du mélange entre l'huile de pression et l'huile d'extraction subissent ensuite un traitement de raffinage plus ou moins poussé selon les débouchés envisagés afin d'éliminer les impuretés, les contaminants éventuels, et de garantir des spécifications physico-chimiques et organoleptiques adaptées aux besoins des utilisateurs en aval (usages alimentaires, techniques, biocarburant).

\section{L'hexane : solvant privilégié pour l'extraction des graines oléagineuses}

L'hexane est aujourd'hui le seul solvant employé industriellement pour l'extraction des huiles végétales. Il a été sélectionné depuis de nombreuses années pour ses propriétés apolaires qui lui confèrent une grande affinité pour les lipides. 


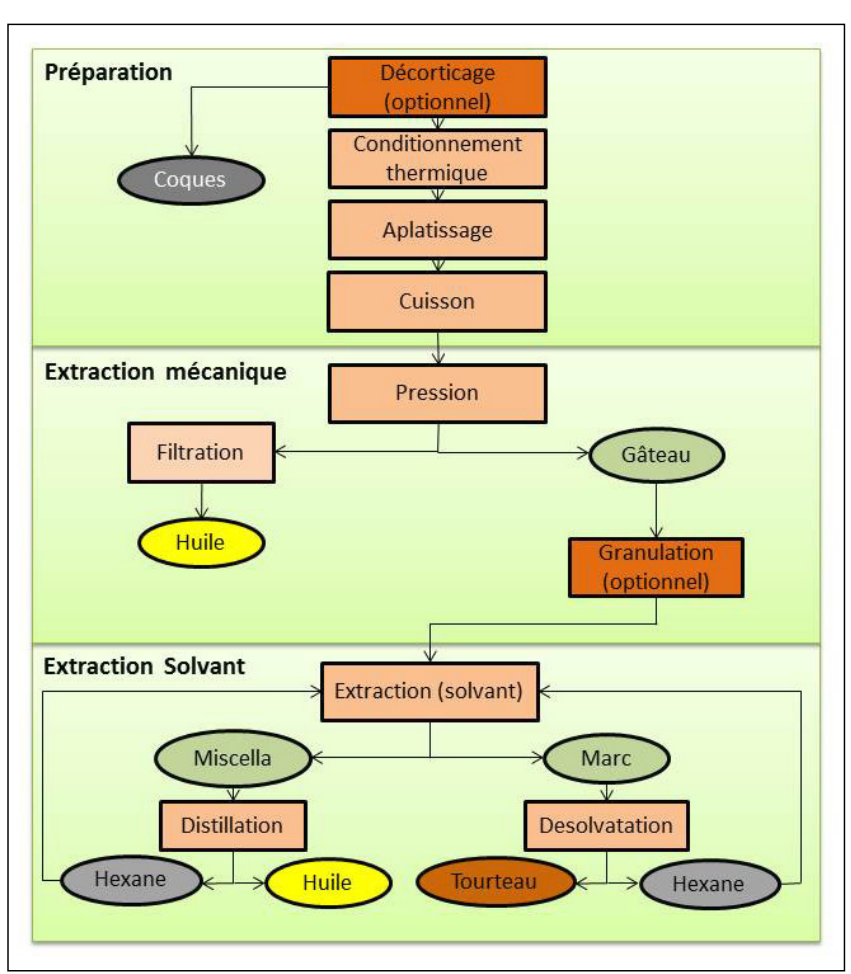

Fig. 1. Opérations unitaires du procédé de trituration de graines oléagineuses.

En outre, il présente l'avantage d'être très sélectif vis-à-vis des huiles et d'avoir une chaleur latente de vaporisation assez faible $(330 \mathrm{~kJ} / \mathrm{kg})$ ce qui permet de l'évaporer facilement, de façon très poussée, et pour un coût énergétique limité. Le recours à l'hexane dans le procédé de trituration des graines oléagineuses, permet d'assurer un rendement d'extraction de l'ordre de $97 \%$ contre seulement $89 \%$ par recours aux seuls moyens mécaniques.

Cependant l'hexane est susceptible d'être remis en question en raison de sa toxicité sur le système nerveux (Galvin, 1997) et de son inflammabilité. C'est une substance dangereuse présentant plusieurs classifications : facilement inflammable $(\mathrm{F})$, reprotoxique de catégorie 3 , nocif $(\mathrm{Xn})$, irritant $(\mathrm{Xi})$ et dangereux pour l'environnement $(\mathrm{N})$. De fait, la toxicité de l'hexane donne obligatoirement lieu à la mise en place de précautions particulières (notamment le travail en zone à risque d'explosions) lors de sa mise en œuvre par une main-d'œuvre industrielle. En effet, une exposition à long terme à l'hexane est susceptible de causer une atteinte du système nerveux central et des troubles de la fertilité. Enfin, rappelons que son utilisation pour l'extraction des huiles et corps gras est réglementée en termes de quantité d'hexane consommé par tonne de graines triturées.

Malgré l'optimisation de ses conditions d'utilisation, la recherche d'alternatives se développe. Les nouvelles voies explorées se situent notamment dans la recherche de solvants de substitution.

En France, l'agence de l'environnement et de la maîtrise de l'énergie (ADEME) a publié un rapport le 21 janvier 2011 analysant la situation de toutes les industries émettrices de composés organiques volatils en vue d'identifier les actions à réaliser pour lever les freins s'opposant à leur substitution (Stavrakakis, 2001). Pour la trituration des graines oléagineuses, il a été établi qu'il n'existait pas de technologie ayant des performances comparables à celle mettant en œuvre de l'hexane et qu'il était préconisé de mettre en place des programmes de R \& D en partenariat entre équipementiers, centres techniques et utilisateurs. Le recours à des solvants moins volatils que l'hexane figure parmi les pistes de recherche suggérées.

\section{Les Agro-solvants: solution de remplacement à l'hexane?}

À l'instar de la raffinerie de pétrole, le concept de bioraffinerie, né dans les années 1980, vise à exploiter les plantes dans leur intégralité pour obtenir différents produits ayant des usages divers : biocarburants, agro-matériaux, polymères, tensioactifs... et solvants (Kamm et Kamm 2004). Les solvants d'origine végétale issus de cette industrie, encore émergente, constituent l'une des catégories de solvants de substitution.

Issus au moins pour partie de matières premières renouvelables, ils présentent l'avantage d'offrir une alternative aux ressources fossiles et un bilan environnemental positif. Ces molécules se distinguent de certains solvants dits « verts », comme les esters dibasiques (Massawe et al., 2008) ou les liquides ioniques (Rogers et Seddon, 2003), possédant de bons profils environnementaux et toxicologiques, mais synthétisés à partir du pétrole.

Les agro-solvants se regroupent en différentes catégories et l'on distingue souvent d'une part ceux issus de la sucrochimie et d'autre part ceux issus de la lipochimie.

\subsection{Les principales sources d'agro-solvants}

On distingue trois types d'agro-solvants classés en fonction de la filière dont ils sont issus (Fig. 2) :

- La filière céréalière/sucrière : les agro-solvants issus de cette filière sont principalement obtenus à partir de la fermentation naturelle des jus sucrés contenus dans les plantes telles que la betterave à sucre, la canne à sucre, le blé, le maïs, etc. L'éthanol d'origine végétale, encore appelé bioéthanol, est issu de la fermentation des sucres contenus dans différentes matières premières agricoles : les plantes saccharifères (betterave sucrière, canne à sucre), les plantes amylacées (blé, maïs) et les ressources lignocellulosiques. En outre, la fermentation du glucose offre de nombreuses possibilités de production de molécules utilisables comme agro-solvants tels que le sorbitol, les esters d'acide lactique (le lactate d'éthyle), les dérivés de l'acide succinique, etc. Enfin, les résidus lignocellulosiques issus de la production de céréales, notamment des pailles, peuvent être exploités pour produire du furfural.

- La filière oléo-protéagineuse : la lipochimie permet de produire des solvants à partir de plantes dont les graines sont riches en huiles végétales, comme le colza, le tournesol, le soja... Les principales molécules d'agro-solvants obtenues sont les esters d'acides gras et les dérivés du glycérol. Les 


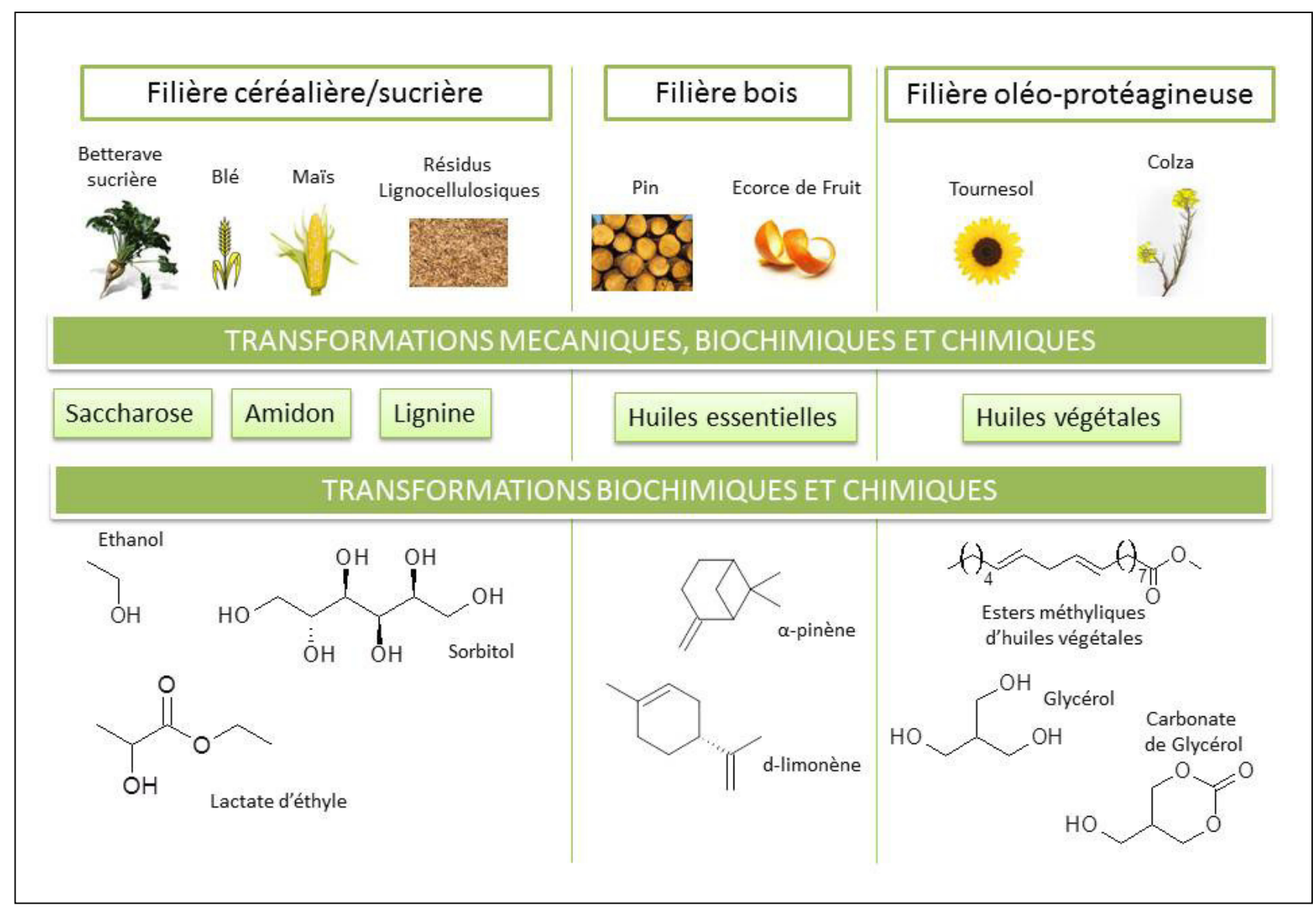

Fig. 2. Agro-solvants issus des grandes filières végétales.

esters d'acides gras sont majoritairement obtenus à partir des triglycérides selon une étape de transestérification dans un alcool (méthanol, éthanol). Cette transformation permet par ailleurs de produire du glycérol qui peut servir de matière première pour la production d'agro-solvants comme, par exemple, le carbonate de glycérol.

- La filière bois : les solvants de cette classe sont produits principalement à partir des conifères tels que le pin ou d'écorces de fruits. Les principaux solvants issus de cette filière sont les hydrocarbures terpéniques de type $\mathrm{C}_{10} \mathrm{H}_{16}$, monocycliques (d-limonène) et bicycliques ( $\alpha$ et $\beta$ pinène). Le d-limonène est produit par distillation à partir des sous-produits générés par l'industrie d'extraction du jus d'orange. L' $\alpha$-pinène et le $\beta$-pinène, peuvent être obtenus par distillation d'oléorésines contenues dans le pin ou encore être extraits des essences de térébenthine après distillation des résines de bois ou des liqueurs noires de papeteries (Meier, 1993).

Parmi les différents agro-solvants cités, les terpènes présentent d'intéressantes propriétés physico-chimiques pour l'extraction des huiles végétales en raison de leur caractère apolaire et de leur faible poids moléculaire.

Pour mener à bien cette démarche de substitution, il convient d'évaluer les propriétés physico-chimiques ainsi que les performances de solubilisation de ces nouvelles molécules, les terpènes, et de les comparer avec celles des solvants de référence, l'hexane.

\subsection{Propriétés physico-chimiques des agro-solvants terpéniques}

Il existe un certain nombre d'outils efficaces, souvent empiriques, permettant de classer ou de sélectionner des solvants pour une application donnée, en fonction de leurs propriétés solvantes et/ou physico-chimiques. Pour estimer et quantifier rapidement le pouvoir solvant et sélectionner un composé approprié pour une application donnée, les paramètres de solubilité de Hansen constituent un guide qualitatif et empirique très utilisé en milieu industriel (Hansen, 1967).

Développée dans les années 1960 pour l'industrie des peintures et vernis, la théorie des paramètres de solubilité de Hansen a depuis été largement utilisée dans de nombreux secteurs d'activité : pharmaceutique, détergence, cosmétique, extraction... La méthode permet, entre autres, de prédire la compatibilité ou l'affinité entre différentes substances chimiques et notamment d'estimer la capacité des agro-solvants à substituer les solvants toxiques d'origine pétrochimique (Mouloungui et al., 2006).

Afin de tenir compte des autres forces intermoléculaires, Hansen a décomposé l'énergie de cohésion totale du système en la somme des énergies de cohésion correspondant aux modes d'interactions principaux rencontrés dans les matériaux organiques courants. Cette décomposition permet ainsi de définir trois paramètres de solubilité : $\delta \mathrm{d}, \delta \mathrm{p}$ et $\delta \mathrm{h}$. 
Tableau 1. Propriétés physico-chimiques du n-hexane, d-limonène et $\alpha$-pinène.

\begin{tabular}{|c|c|c|c|}
\hline Solvant & $n$-Hexane & $d$-Limonène & $\alpha$-Pinène \\
\hline \multicolumn{4}{|l|}{ Structure Chimique } \\
\hline Masse moléculaire (g/mol) & 86.18 & 136.23 & 136.24 \\
\hline Densité $25^{\circ} \mathrm{C}\left(\mathrm{g} / \mathrm{cm}^{3}\right)$ & 0.675 & 0.834 & 0.879 \\
\hline Flash point $\left({ }^{\circ} \mathrm{C}\right)$ & -23 & 48.3 & 32 \\
\hline Point d'ébullition, $760 \mathrm{~mm} \mathrm{Hg}\left({ }^{\circ} \mathrm{C}\right)$ & 69 & 176 & 155 \\
\hline Viscosité, $25^{\circ} \mathrm{C}(\mathrm{Cp})$ & 0.31 & 0.83 & 1.32 \\
\hline Tension de surface, $25^{\circ} \mathrm{C}(\mathrm{mN} / \mathrm{m})$ & 20.3 & 25.8 & 25.3 \\
\hline Constante diélectrique, $20^{\circ} \mathrm{C}$ & 1.87 & 2.44 & 2.58 \\
\hline Paramètres de solubilité de Hansen $\delta^{\mathrm{a}}\left(\mathrm{cal}^{1 / 2} \mathrm{~cm}^{-3 / 2}\right)$ & 7.3 & 8 & 7.9 \\
\hline$\delta_{d}$ & 7.3 & 8 & 7.6 \\
\hline$\delta_{p}$ & 0 & 0.1 & 2.1 \\
\hline$\delta_{\mathrm{h}}$ & 0 & 0.1 & 0 \\
\hline
\end{tabular}

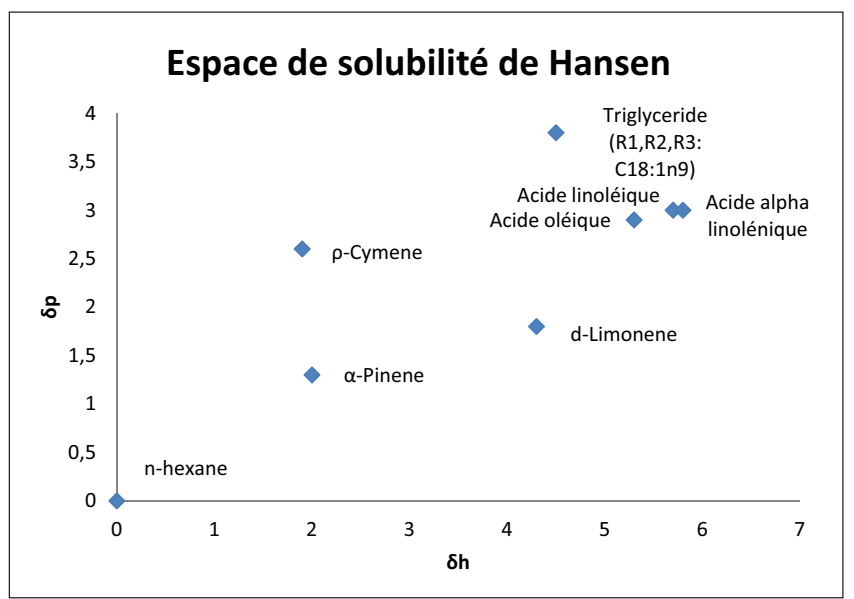

Fig. 3. Représentation des solvants terpéniques, de l'hexane et des constituants majeurs des huiles végétales dans l'espace de solubilité $\delta \mathrm{p} v$ s. $\delta \mathrm{h}$.

En d'autres termes, pour que deux substances soient miscibles, il est nécessaire que leurs trois paramètres de solubilité soient identiques ou très proches. De façon représentative, les paramètres de Hansen permettent d'accéder à un espace de solubilité tridimensionnel. Cependant, des trois paramètres de solubilité, le paramètre $\delta$ d est celui qui présente le moins de variations. C'est pourquoi, généralement, pour situer les composés, on préfère utiliser une représentation bidimensionnelle $\delta \mathrm{p}$ $v s$. $\delta \mathrm{h}$, plus facile à visualiser qu'un espace à trois dimensions.

\subsection{Exemples d'applications}

Une étude des paramètres de solubilité de deux terpènes, le $\mathrm{d}$-limonène et l' $\alpha$-pinène, par la méthode d'Hansen a permis de mettre en évidence une certaine similarité de ces derniers avec l'hexane (Tab. 1). Les valeurs des trois termes descriptifs sont semblables; cela implique ainsi un comportement similaire des deux solvants terpéniques et du n-hexane en solution. De ce point de vue, les terpènes sont aussi efficaces que le n-hexane pour dissoudre les huiles. Cependant, en considérant la valeur de la constante diélectrique, les terpènes semblent plus polaires et possèdent un pouvoir dissociatif plus élevé que le n-hexane. D'un point de vue de la sécurité, les terpènes possèdent des points d'éclair supérieur à celui du n-hexane; ils sont donc moins inflammables et dangereux.

Les premiers travaux sur l'extraction d'huile végétale à l'aide du $d$-limonène ont été réalisés par Liu et Mamidipally (2004 ; 2005). Les auteurs ont étudié et proposé, avec de bons rendements, la possible extraction d'huile de son de riz, en vue de son application à l'échelle industrielle. Les caractéristiques de qualité (acides gras libres, couleur, teneur en phospholipides) de l'huile brute extraite, dans les conditions optimales, selon les deux solvants, n-hexane et d-limonène, se sont avérées comparables.

Les laboratoires GREEN ont développé un nouveau dispositif d'extraction de matières grasses par micro-ondes appelé : «Soxhlet assisté par micro-ondes » (Virot et al., 2007). Depuis 130 ans, le Soxhlet a été la technique de laboratoire la plus commune pour l'extraction des graisses et huiles à partir de matrices alimentaires. Cependant, les inconvénients majeurs de cette méthode résident dans le temps d'extraction long, la consommation d'énergie, et l'utilisation de grandes quantités de solvants pétroliers. Ce nouveau système conduit à des résultats équivalents à ceux obtenus avec le n-hexane utilisé selon des normes standardisées. Ce procédé, dans lequel $90 \%$ du solvant est recyclé, a réduit le temps exhaustif de $8 \mathrm{~h}$ de traitement dans la méthode conventionnelle à seulement 32 min. Allant plus loin, Virot et al., (2008) ont proposé 


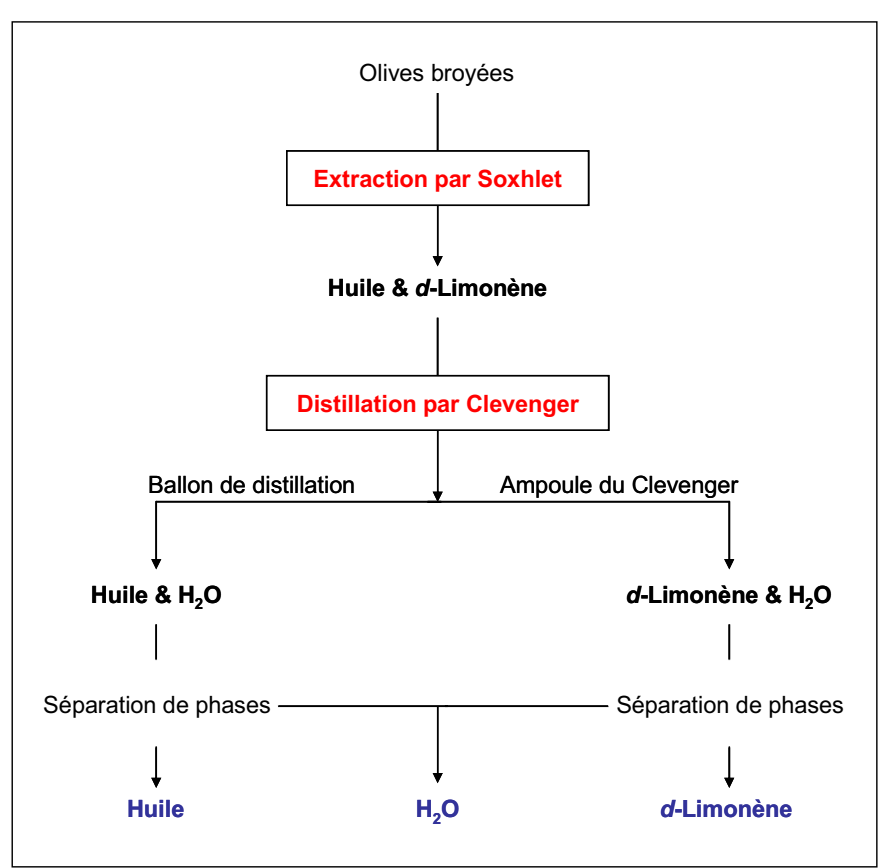

Fig. 4. Procédure utilisée pour éliminer le d-limonène (cas des olives).

la combinaison du «Soxhlet assisté par micro-ondes » et du dlimonène comme agro-solvant. L'étape d'extraction est suivie d'une distillation par micro-ondes utilisant le système de type Clevenger. Le processus proposé a été appliqué avec succès à l'extraction de l'huile d'olive. Afin de surmonter le problème du point d'ébullition élevé exigé par le d-limonène pour être recyclé, les auteurs ont proposé une approche innovante en capitalisant sur le concept de distillation azéotropique d'huiles essentielles (entraînement à la vapeur ou hydrodistillation) à l'aide d'un dispositif de type Clevenger. Après l'étape d'extraction au Soxhlet avec le d-limonène, de l'eau distillée a été ajoutée au mélange composé d'huile extraite et de d-limonène. Après distillation de l'eau azéotropique autour de $100{ }^{\circ} \mathrm{C}$ avec un système de type Clevenger, le d-limonène et l'huile extraite ont été récupérés séparément (Fig. 4).

Les résultats obtenus par les deux techniques d'extraction ont montré que le rendement d'extraction obtenu avec le d-limonène a été légèrement supérieur à celui obtenu avec le n-hexane. Cette différence s'explique par le caractère légèrement moins polaire du n-hexane vis-à-vis du d-limonène et d'un pouvoir solvant plus marqué envers les triglycérides de ce dernier.

Les résultats des analyses des esters méthyliques d'acides gras (EMAG) par CG-SM sont conformes à ceux obtenus dans la littérature (Di Bella et al., 2007) en termes d'analyse qualitative où aucune différence significative entre les deux méthodes n'a été détectée $(P>0,05)$ (Tab. 2). Néanmoins, on peut noter un taux plus élevé d'acides gras libres, de l'indice de peroxyde et des diènes conjugués dans les extraits obtenus avec le d-limonène. Ces résultats ont révélé la présence de traces d'oxydation, qui peut être attribuée à la température plus élevée, utilisée pour extraire l'huile.

Plus récemment, l'équipe GREEN a démontré le potentiel des solvants terpéniques comme alternative aux solvants
Tableau 2. Comparaison des huiles extraites à partir d'olives par n-hexane et d-limonène.

\begin{tabular}{lccc}
\hline \multicolumn{1}{c}{ Acides gras } & n-hexane $(\%)$ & d-limonène (\%) \\
\hline Palmitique & $\mathrm{C} 16: 0$ & 12,7 & 14,1 \\
Palmitoléique & $\mathrm{C} 16: 1$ & 1,0 & 1,1 \\
Margaroléique & $\mathrm{C} 17: 1$ & 0,2 & 0,3 \\
Stéarique & $\mathrm{C} 18: 0$ & 2,2 & 2,4 \\
Oléique & $\mathrm{C} 18: 1$ & 72,0 & 71,7 \\
Linoléique & $\mathrm{C} 18: 2$ & 10,0 & 8,6 \\
Linolénique & $\mathrm{C} 18: 3$ & 0,8 & 0,4 \\
Eicosanoique & $\mathrm{C} 20: 0$ & 0,5 & 0,4 \\
Gadoléique & $\mathrm{C} 20: 1$ & 0,3 & 0,4 \\
\hline Béhénique & $\mathrm{C} 22: 0$ & 0,2 & 0,1 \\
\hline$\sum \boldsymbol{S F A s}$ & & 15,62 & 17,36 \\
$\sum \boldsymbol{M U F A s}$ & & 73,53 & 73,44 \\
$\sum \boldsymbol{P} \boldsymbol{P} \boldsymbol{F A s}$ & & 10,77 & 9,02 \\
\hline Rendements $(\%)$ & & $40,3 \pm 0,7$ & $48,6 \pm 2,2$ \\
\hline
\end{tabular}

SFAs : Acides gras saturés, MUFAs : Acides gras mono-insaturés, PUFAs : Acides gras poly-insaturés, tr. : traces, nd. : non détecté.

pétroliers, tels que le n-hexane, pour l'extraction d'huile de micro-algues (Dejoye Tanzi et al., 2012). Les huiles extraites ont été comparées d'un point de vue qualitatif et quantitatif. Aucune différence significative n'a été observée entre chaque extrait.

\section{Freins et verrous à lever pour le développement des agro-solvants}

Il existe encore de nombreux freins et verrous à lever avant d'envisager un développement industriel de ces nouveaux solvants pour une application à l'extraction des huiles végétales. Le premier est d'ordre réglementaire avec la directive 2009/32 du parlement européen qui limite la liste des solvants utilisables pour l'extraction des denrées alimentaires. Par contre, il n'existe pas à notre connaissance d'interdiction formelle concernant des tourteaux qui seraient produits avec d'autres solvants que ceux qui figurent dans la directive «solvants". Cette étape réglementaire devrait toutefois pouvoir être franchie dans la mesure où les agro-solvants sont généralement peu dangereux. Par exemple, le d-limonène est classé GRAS (Generaly Recognized as Safe) par l'administration Etasunienne des aliments et des drogues (USFDA, 1997). La recommandation commune OMS / FAO du Codex Alimentarius concernant la dose journalière admissible est de $0,075 \mathrm{mg} / \mathrm{kg}$ de masse corporelle pour le limonène en tant qu'additif et $1,5 \mathrm{mg} / \mathrm{kg}$ de masse corporelle pour le limonène total (Codex Alimentarius, 1993). Si on utilise ce dernier ratio pour estimer une dose a priori admissible dans l'alimentation de monogastriques, l'ordre de grandeur du limonène résiduel dans les tourteaux ne devrait pas dépasser $200 \mathrm{mg} / \mathrm{kg}$.

Un autre verrou à lever est le recyclage du solvant. En effet, pour entraîner efficacement le solvant et atteindre une teneur résiduelle de l'ordre de celle qui est évoquée ci-dessus, une injection de vapeur importante devra avoir lieu dans le désolvanteur et le temps de séjour devra être prolongé. La technologie 
de dé-solvantation devra donc être adaptée pour réduire la rétention en solvant du marc d'une part et pour travailler sous pression réduite d'autre part en vue de réduire la température de travail.

D'autres inconnues doivent faire l'objet d'investigations. Ainsi, la stabilité et la non réactivité des agro-solvants au cours de nombreux cycles extraction/régénération demande à être vérifiée. La percolation du solvant dans un extracteur industriel pourrait s'avérer moins aisée que dans le cas de l'hexane si celui-ci parvenait à faire gonfler le gâteau de pression. Certains polymères utilisés comme joints ou dans des parties de tuyauteries pourraient avoir une mauvaise tenue dans ces nouveaux solvants. Des composés mineurs pourraient se retrouver présents dans les huiles et poser ensuite des problèmes de raffinage.

\section{Conclusion}

L'épuisement progressif des ressources pétrolières, la réglementation européenne $\mathrm{REACH}$, et le souhait du consommateur de disposer de produits respectueux de l'environnement donnent à la chimie durable le bien-fondé de son développement. Dans le contexte actuel de substitution des solvants toxiques d'origine pétrochimique, plusieurs alternatives industrielles sont envisageables et offrent d'une part, l'opportunité de relever de nouveaux challenges technologiques et d'autre part, d'exploiter de nouvelles sources de matières premières. Parmi les solutions de remplacement des solvants pétrochimiques, l'utilisation des agro-solvants, obtenus par au moins une partie de matières premières renouvelables tels que les plantes sucrières, les plantes oléagineuses..., représentent une voie de substitution intéressante, déjà développée pour de nombreuses applications industrielles. Ainsi, l'utilisation de limonène, issu de la filière orange, présente un intérêt en termes de sécurité et de non toxicité. Elle peut être envisagée dans l'extraction en laboratoire. Son utilisation industrielle présente a priori une difficulté économique.

Globalement, on peut penser que la plupart des verrous pour l'utilisation des agro-solvants pour l'extraction des graines oléagineuses pourront être levés grâce à des travaux de recherche et développement. En revanche, il est probable que le coût final de la technologie sera supérieur à celui du procédé à l'hexane, ce qui ne devrait pas inciter les triturateurs à choisir volontairement d'effectuer le saut technologique sans incitation forte des pouvoirs publics, qu'elle soit de nature financière ou réglementaire. Il reste néanmoins une possibilité qui consisterait à produire une huile améliorée, ce qui la rendrait désirable pour le consommateur et permettrait de sortir du dilemme par le haut.

\section{Références}

Codex Alimentarius. 1993. Programme mixte FAO/OMS sur les normes alimentaires, Vingtième session, Genève, 28 juin-7 juillet 1993.

Dejoye Tanzi C, Albert Vian M, Ginies C, Elmaataoui M, Chemat F. 2012. Terpenes as Green Solvents for Extraction of Oil from Microalgae. Molecules 17 : 8196-8205.

Di Bella G, Maisano R, La Pera L, Lo Turco V, Salvo F, Dugo G. 2007. Statistical characterization of sicilian olive oils from the Peloritana and Maghrebian zones according to the fatty acid profile. J. Agric. Food Chem. 55 : 6568-6574.

Galvin JB. 1997. Toxicity data for commercial hexane and hexane isomers. Technology and solvents for extracting oilseeds and nonpetroleum Oils. AOCS Press, pp. 75-85.

Hansen CM. 1967. The three dimensional solubility parameter - key to paint component affinities II. - Dyes, emulsifiers, mutual solubility and compatibility, and pigments. J. Paint Technol. 39 : 505-510.

Kamm B, Kamm M. 2004. Principles of biorefineries. Appl. Microbiol. Biotechnol. 64 : 137-145.

Liu SX, Mamidipally PK. 2005. Quality comparison of rice bran oil extracted with d-limonene and hexane. Cereal Chem. 82 : 209-215.

Mamidipally PK, Liu SX. 2004. First approach on rice bran oil extraction using limonene. Eur. J. Lipid Sci. Technol. 106 : 122-125.

Massawe E, Geiser K, Ellenbecker M, Marshall J. 2008. Technical performance evaluation of the potential biobased floor strippers. J. Clean. Prod. $16: 12-21$.

Meier GJ. 1993. Non-aqueous cleaning solvent substitution. In: National technology transfer conference and exposition (4th), Anaheim, CA, United States Department of Energy, Washington, DC. 7-9 December, pp. 223-231.

Mouloungui Z, Pelet S, Eychenne V, Mateo S. 2006. Lipochimie. In : Colonna P, La chimie verte. Paris : Lavoisier, Tec \& Doc, 2006.

Rogers RD, Seddon KR. 2003. Ionics liquids as green solvents: Progress and prospects. ACS Symposium Series 856.

Stavrakakis C., 2001, Freins à la substitution des composés organiques volatils dans les procédés industriels. Rapport final. Étude réalisée pour le compte de l'ADEME par le CITEPA (ref 822). Disponible en ligne sur le site de l'ADEME (http://www2.ademe.fr).

USFDA, 1997, Eligibility for classification as generally recognized as safe (GRAS) Code of Federal Regulation Title 21, Section 170.30. United States Department of Health and Human Service, Food and Drug Administration, Washington, DC.

Virot M, Tomao V, Colnagui G, Visinoni F, Chemat F. 2007. New microwave-integrated Soxhlet extraction. An advantageous tool for the extraction of lipids from food products. J. Chromatogr. A $1174: 138-144$.

Virot M, Tomao V, Ginies C, Visinoni F, Chemat F. 2008. Microwaveintegrated extraction of total fats and oils. J. Chromatogr. A 1196 : $57-64$.

Cite this article as: Frederic Fine, Maryline Abert Vian, Anne-Sylvie Fabiano Tixier, Patrick Carre, Xavier Pages, Farid Chemat. Les agro-solvants pour l'extraction des huiles végétales issues de graines oléagineuses. OCL 2013, 20(5) A502. 